\title{
Demonstration of epitaxial growth of strain-relaxed GaN films on graphene/SiC substrates for long wavelength light-emitting diodes
}

Ye Yu' ${ }^{1}$, Tao Wang ${ }^{2}$, Xiufang Chen ${ }^{3}$, Lidong Zhang ${ }^{1}$, Yang Wang ${ }^{1}$, Yunfei Niu', Jiaqi Y ${ }^{1}$, Haotian Ma', Xiaomeng Li ${ }^{3}$, Fang Liư ${ }^{4}$, Gaoqiang Deng ${ }^{1}$, Zhifeng Shi', Baolin Zhang ${ }^{1}$, Xinqiang Wang $\mathbb{1}^{4}$ and Yuantao Zhang ${ }^{1}$

\begin{abstract}
Strain modulation is crucial for heteroepitaxy such as GaN on foreign substrates. Here, the epitaxy of strain-relaxed GaN films on graphene/SiC substrates by metal-organic chemical vapor deposition is demonstrated. Graphene was directly prepared on $\mathrm{SiC}$ substrates by thermal decomposition. Its pre-treatment with nitrogen-plasma can introduce $\mathrm{C}-\mathrm{N}$ dangling bonds, which provides nucleation sites for subsequent epitaxial growth. The scanning transmission electron microscopy measurements confirm that part of graphene surface was etched by nitrogen-plasma. We study the growth behavior on different areas of graphene surface after pre-treatment, and propose a growth model to explain the epitaxial growth mechanism of GaN films on graphene. Significantly, graphene is found to be effective to reduce the biaxial stress in GaN films and the strain relaxation improves indium-atom incorporation in InGaN/GaN multiple quantum wells (MQWs) active region, which results in the obvious red-shift of light-emitting wavelength of InGaN/ GaN MQWs. This work opens up a new way for the fabrication of GaN-based long wavelength light-emitting diodes.
\end{abstract}

\section{Introduction}

Due to direct wide bandgap and high stability, group IIInitride semiconductors have been widely used in lightemitting diodes (LEDs), laser diodes and photodetectors ${ }^{1-3}$. Currently, the high cost of native substrates makes group IIInitrides usually grow on foreign substrates, such as sapphire, $\mathrm{SiC}$, and $\mathrm{Si}^{4-6}$. However, there are lattice and thermal mismatches between nitride films and foreign substrates, leading to the formation of high-density point defects, threading dislocations, and residual stress in the prepared films and devices, which degrade the working lifetime and efficiency of GaN-based optoelectronic devices ${ }^{7-9}$.

\footnotetext{
Correspondence: Xiufang Chen (cxf@sdu.edu.cn) or

Yuantao Zhang (zhangyt@jlu.edu.cn)

'State Key Laboratory of Integrated Optoelectronics, College of Electronic Science and Engineering, Jilin University, Qianjin Street 2699, Changchun 130012, China

2Electron Microscopy Laboratory, School of Physics, Peking University, Beijing 100871, China

Full list of author information is available at the end of the article

These authors contributed equally: Ye Yu, Tao Wang
}

Recently, GaN films grown on graphene and other twodimensional materials have attracted extensive attention $^{10-13}$. The epitaxial growth of $\mathrm{GaN}$ on graphene forms weak covalent bonds at the interface between graphene and epitaxial layer, which makes the lattice at the interface produce no large strain as the traditional heteroepitaxy, and the stress in films greatly reduced ${ }^{14}$. This provides a new path for heteroepitaxial growth of $\mathrm{GaN}$ films. In addition, the weak force between the epitaxial layer and graphene can make the epitaxial layer be easily released from the substrate and transferred to other materials, realizing some novel device applications, such as flexible devices ${ }^{15,16}$. However, the lack of dangling bonds on graphene suppresses the GaN nucleation, limiting the growth of continuous and smooth GaN films on graphene ${ }^{17}$. To solve this problem, some researches have achieved the epitaxial growth of nitrides on graphene by introducing interfacial buffers or defects on graphene. For instance, Chung et al. realized the epitaxial growth of the GaN-based LED structures on graphene/sapphire

\section{(c) The Author(s) 2021}

(c) Open Access This article is licensed under a Creative Commons Attribution 4.0 International License, which permits use, sharing, adaptation, distribution and reproduction c. in any medium or format, as long as you give appropriate credit to the original author(s) and the source, provide a link to the Creative Commons license, and indicate if changes were made. The images or other third party material in this article are included in the article's Creative Commons license, unless indicated otherwise in a credit line to the material. If material is not included in the article's Creative Commons license and your intended use is not permitted by statutory regulation or exceeds the permitted use, you will need to obtain permission directly from the copyright holder. To view a copy of this license, visit http://creativecommons.org/licenses/by/4.0/. 

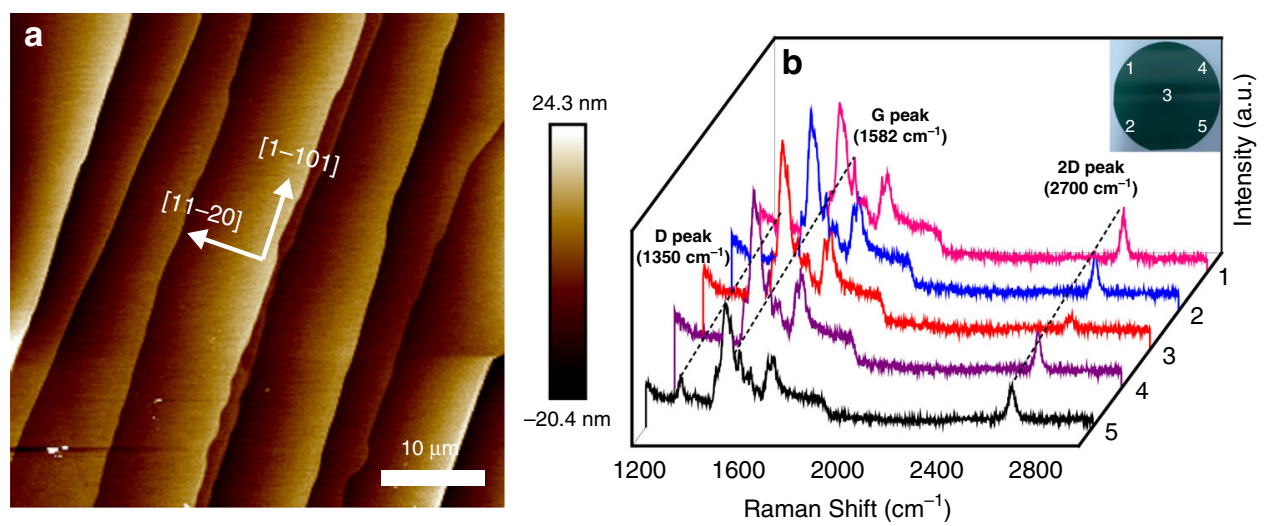

Fig. 1 Characterizations of graphene/SiC substrates. a AFM image of graphene/SiC substrate, showing the step-like structure. $\mathbf{b}$ Raman spectra of five measurement positions on 2 " graphene/SiC substrate. The inset shows the photograph of as-grown 2 " graphene/SiC substrate and the measured positions

substrates by using of high-density, vertically distributed $\mathrm{ZnO}$ nanowalls as the interfacial buffer ${ }^{10}$. Besides, Chen et al. reported the epitaxial growth of AlN films on graphene by nitrogen-plasma pre-treatment ${ }^{18}$. Although there are a few reports on the epitaxial growth of $\mathrm{GaN}$ on graphene, the epitaxial growth mechanism of $\mathrm{GaN}$ on graphene is still not clear enough, and the role of graphene in the $\mathrm{GaN}$ epitaxial growth process is unclear.

Here, we successfully grew a strain-relaxed GaN film on graphene/SiC substrates by metal-organic chemical vapor deposition (MOCVD). Graphene was directly prepared on $\mathrm{SiC}$ substrates by thermal decomposition. The nitrogenplasma pre-treatment was performed on graphene surface, introducing the $\mathrm{C}-\mathrm{N}$-related dangling bonds, which promoted the subsequent epitaxial growth of $\mathrm{GaN}$ films. The scanning transmission electron microscopy (STEM) measurements reveal that part of graphene surface was etched by nitrogen-plasma pre-treatment. The growth behavior on different areas of nitrogen-plasma-treated graphene was studied, and then a growth model was proposed based on the STEM results to explain the epitaxial growth mechanism of $\mathrm{GaN}$ on graphene. It is worth noting that, the graphene is found to be effective to reduce the biaxial stress in GaN films. Then, we prepared InGaN/GaN multiple quantum wells (MQWs) on the strain-relaxed GaN films. The strain relaxation in GaN films leads to the improvement of indium-atom incorporation efficiency in the MQWs, resulting in the obvious red-shift of light-emitting wavelength. Our work enhances the understanding of epitaxial growth of nitrides on graphene, and provides a new way for the fabrication of nitride long wavelength GaN-based LEDs.

\section{Results}

To avoid the inevitable pollution, mechanical damage, and size limitation of graphene during the transfer process, graphene was directly prepared on $\mathrm{Si}$-face $4 \mathrm{H}$ $\mathrm{SiC}$ substrates by thermal decomposition. Figure 1a shows surface morphology of the prepared graphene on $\mathrm{SiC}$ substrate measured by the atomic force microscopy (AFM). The AFM image presents a step-like surface morphology with a consistent direction, uniform width and height. The measured terrace width and step height are about $5-10 \mu \mathrm{m}$ and $10-15 \mathrm{~nm}$, respectively. To characterize the uniformity of the graphene on $\mathrm{SiC}$ substrate, we measured Raman spectra at five different positions on a $2^{\prime \prime}$ diameter graphene/SiC substrate, as shown in Fig. 1b. The inset displays the photograph of the $2^{\prime \prime}$ graphene/SiC substrate and the measured positions. The characteristic G peak $\left(\sim 1582 \mathrm{~cm}^{-1}\right)$ and 2D peak $\left(\sim 2700 \mathrm{~cm}^{-1}\right)$ of graphene can be seen in Raman spectra of five measured positions, which indicates that the prepared graphene on $\mathrm{SiC}$ substrate by thermal decomposition has good uniformity ${ }^{19,20}$. In addition, we calculated the intensity ratio of the characteristic $2 \mathrm{D}$-to-G peak $\left(I_{2 \mathrm{D}} /\right.$ $I_{\mathrm{G}}$ ) of graphene, and the intensity ratio of the defectrelated D peak $\left(\sim 1350 \mathrm{~cm}^{-1}\right)$ to the characteristic $G$ peak $\left(I_{\mathrm{D}} / I_{\mathrm{G}}\right)$ of that. The results show that the prepared graphene on $\mathrm{SiC}$ substrate is the multilayer one with good quality (see Fig. S1) ${ }^{21}$.

GaN films were grown on graphene/SiC substrates using MOCVD. The lack of dangling bonds on graphene surface is not conducive to the nucleation growth of $\mathrm{GaN}^{17}$. The as-grown $\mathrm{GaN}$ on graphene cannot form a continuous film, and the surface morphology presents a discrete island-like distribution with independent nucleation, as shown in Fig. 2a. In order to enhance the nucleation ability of nitrides on graphene, we performed nitrogen-plasma pre-treatment on graphene before the epitaxial growth, and optimized the buffer layer in the epitaxial structure (see Fig. S2). Based on the above efforts, the continuous and smooth GaN films were obtained on graphene by 

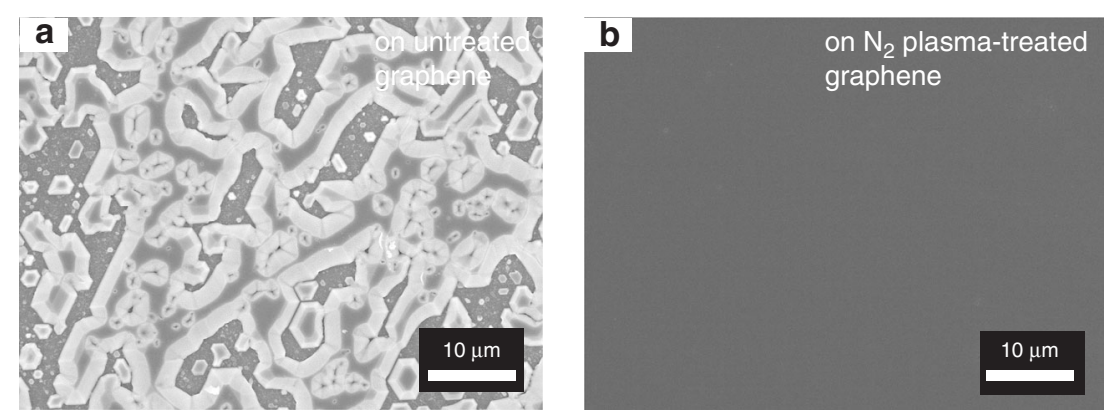

Fig. 2 Surface morphology of GaN on graphene/SiC. SEM images of GaN on: a untreated graphene, and $\mathbf{b}$ nitrogen-plasma-treated graphene
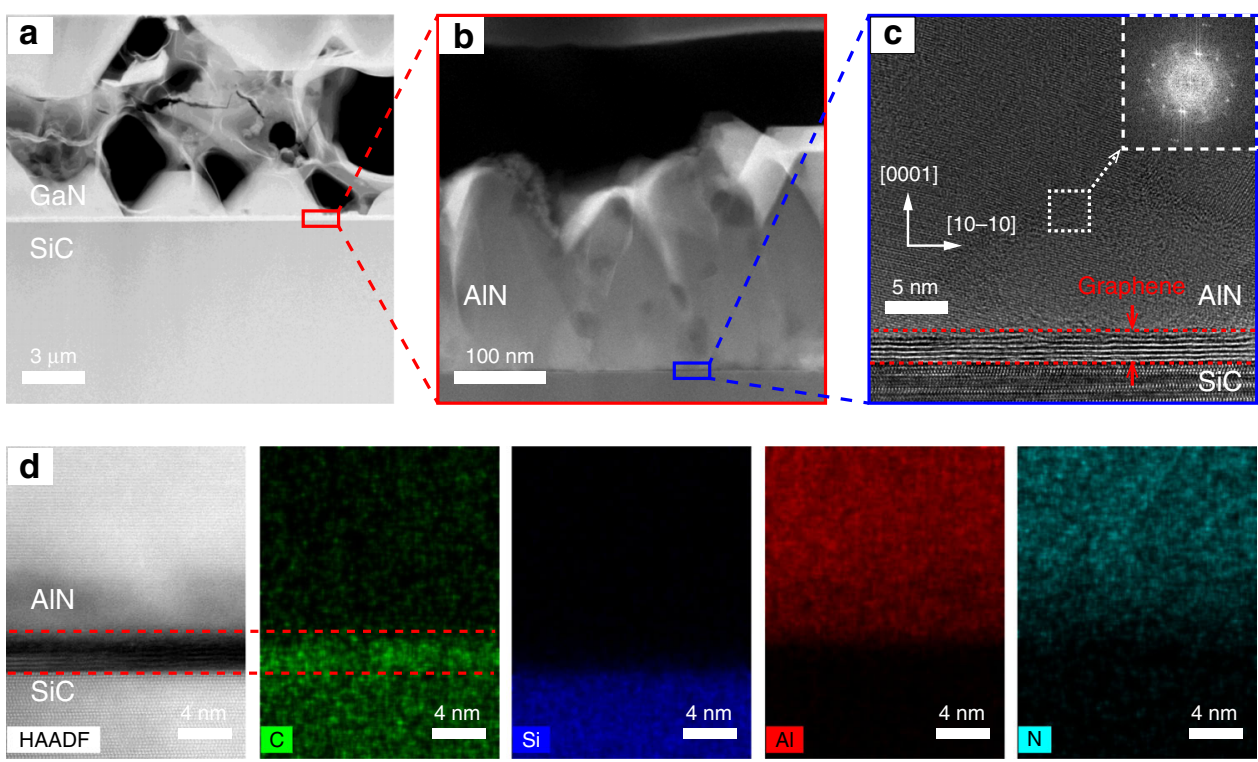

Fig. 3 HAADF-STEM analysis of GaN on untreated graphene/SiC. a Cross-sectional HAADF-STEM image of GaN on untreated graphene. b Crosssectional HAADF-STEM image of the interface area marked with a red rectangle frame in (a). c Cross-sectional HRTEM image of the interface area marked with a blue rectangle frame in (b). The multilayer graphene at the interface is clearly visible. The inset in (c) shows the SAED pattern of AIN in the white square region. $\mathbf{d}$ EDS mappings of elemental $\mathrm{C}, \mathrm{Si}, \mathrm{Al}$, and $\mathrm{N}$ at the interface of AIN/graphene/SiC

using low- and high-temperature AlN buffers, as shown in Fig. 2b.

Cross-sectional high-angle annular dark-field STEM (HAADF-STEM) measurements were performed on the as-grown GaN on untreated graphene. As shown in Fig. $3 \mathrm{a}$, the epitaxial structure is composed of $\mathrm{GaN}$ islands, AlN buffer and graphene/SiC substrate on untreated graphene, verifying that $\mathrm{GaN}$ on untreated graphene shows the discrete islands growth. Figure $3 b$ shows the HAADF-STEM image of the interface region marked with a red rectangular frame in Fig. 3a. It can be seen that the AlN buffer grown on graphene is not completely merged, and there are no $\mathrm{GaN}$ nucleation islands above the AlN buffer, indicating that $\mathrm{GaN}$ nucleation islands are distributed. Figure 3c shows the cross-sectional high-resolution transmission electron microscopy (HRTEM) image of the interface region marked with a blue rectangular frame in Fig. 3b, and the multilayer graphene at the interface can be observed clearly. The inset of Fig. 3c shows the selected area electron diffraction (SAED) pattern of the AlN buffer in the white square region in Fig. 3c. This result indicates that the AlN buffer grown on graphene is polycrystalline. Multilayer graphene can effectively screen the lattice potential field from $\mathrm{SiC}$ substrate, resulting in the forming of the polycrystalline AlN buffer ${ }^{22}$. The SEM and X-ray diffraction (XRD) measurement results also confirm that AlN buffer is polycrystalline (see Fig. S3). The interface elemental components were analyzed by energy dispersive spectroscopy (EDS) mapping. Figure 3d shows the EDS mapping images of different elements at $\mathrm{AlN} /$ graphene/SiC interface. The distributions of $\mathrm{C}, \mathrm{Si}, \mathrm{Al}$, and $\mathrm{N}$ elements 

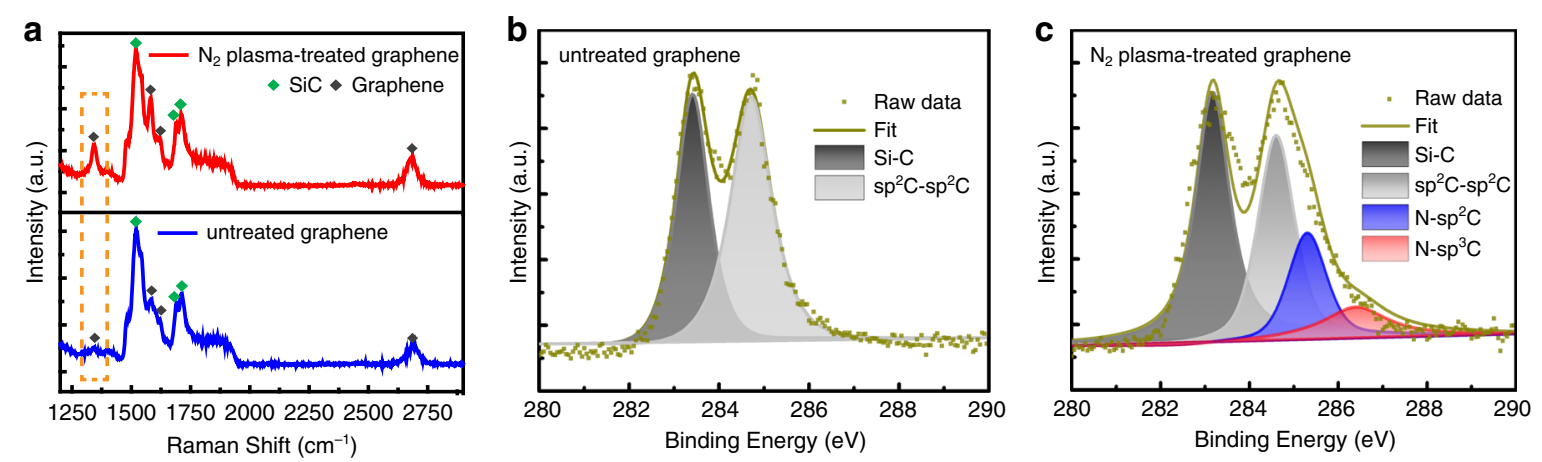

Fig. 4 Raman and XPS analyses of graphene before and after nitrogen-plasma pre-treatment. a Raman spectra of untreated graphene (blue) and nitrogen-plasma-treated graphene (red). The green and black diamond blocks correspond to the characteristic peaks of SiC and graphene, respectively. The orange dashed region shows a significant increase in the intensity of D peak. XPS spectra with C 1s of: $\mathbf{b}$ untreated graphene, and c nitrogen-plasma-treated graphene. XPS results show that nitrogen-plasma pre-treatment introduces $\mathrm{C}-\mathrm{N}$ bonds on graphene surface: $\mathrm{N}-\mathrm{sp}^{2} \mathrm{C}$ bonds $(\sim 285.5 \mathrm{eV})$ and $\mathrm{N}-\mathrm{sp}^{3} \mathrm{C}$ bonds $(\sim 286.5 \mathrm{eV})$

confirm that the graphene still steadily exists after AlN epitaxy, indicating that the graphene is not damaged under high temperature and hydrogen atmosphere of MOCVD system, and still maintains its integrity.

The Raman spectra in Fig. 4a show that the D peak intensity of nitrogen-plasma-treated graphene is greatly increased compared with untreated one, which indicates that defects are introduced on graphene surface by nitrogen-plasma pre-treatment. After that treatment, the $I_{\mathrm{D}} / I_{\mathrm{G}}$ ratio of the graphene in Raman spectra increases from 0.3 to 0.5 . The defect density in graphene can be calculated based on the $I_{\mathrm{D}} / I_{\mathrm{G}}$ ratio, and it increases from $9.0 \times 10^{10} \mathrm{~cm}^{-2}$ to $2.0 \times 10^{11} \mathrm{~cm}^{-223}$. In order to further study the effect of nitrogen-plasma pre-treatment, X-ray photoelectron spectroscopy (XPS) measurements were conducted for untreated and nitrogen-plasma-treated graphene. Figure $4 \mathrm{~b}$ shows the XPS spectrum of $\mathrm{C} 1 \mathrm{~s}$ peak of untreated graphene, which consists of $\mathrm{Si}-\mathrm{C}$ bond $(\sim 283.2 \mathrm{eV})$ from $\mathrm{SiC}$ substrate and $\mathrm{sp}^{2} \mathrm{C}-\mathrm{sp}^{2} \mathrm{C}$ bond $(\sim 284.6 \mathrm{eV})$ from graphene ${ }^{24}$. After nitrogen-plasma pretreatment, two different kinds of $\mathrm{C}-\mathrm{N}$ bonding configurations appeared in $\mathrm{C} 1 \mathrm{~s}$ peak, corresponding to $\mathrm{N}-\mathrm{sp}^{2} \mathrm{C}$ bond $(\sim 285.5 \mathrm{eV})$ and $\mathrm{N}-\mathrm{sp}^{3} \mathrm{C}$ bond $(\sim 286.5 \mathrm{eV})$, as shown in Fig. $4 \mathrm{c}^{25}$. Since the pyrrolic $\mathrm{N}$ atom in $\mathrm{N}-\mathrm{sp}^{3} \mathrm{C}$ bond is more reactive than the pyridinic $\mathrm{N}$ atom in $\mathrm{N}-\mathrm{sp}^{2} \mathrm{C}$ bond ${ }^{11,18}, \mathrm{~N}-\mathrm{sp}^{3} \mathrm{C}$ bond can provide more nucleation sites for subsequent epitaxial growth of GaN films.

Figure 5a shows the cross-sectional HAADF-STEM image of $\mathrm{GaN}$ on nitrogen-plasma-treated graphene. The epitaxial structure composed of GaN film, AlN buffer and graphene/SiC substrate can be observed clearly. Figure $5 \mathrm{~b}$ shows the cross-sectional HAADF-STEM image of the interface region marked with a red rectangular frame in Fig. 5a. It is worth noting that part of graphene was etched after nitrogen-plasma pre-treatment. Among them, the dark part indicated by the red arrow is the unetched graphene region, and the light part indicated by the blue arrow is the etched graphene region. The SAED pattern in Fig. $5 \mathrm{c}$ indicates that the AlN buffer on the unetched graphene region is still polycrystalline, while the AlN buffer on the etched graphene region shows a single crystalline with hexagonal wurtzite structure, as shown in Fig. 5d. Single crystalline AlN plays a dominant role during the subsequent epitaxial growth, which facilitates the epitaxial growth of $\mathrm{GaN}$ films. Figure 5e shows the integrated differential phase contrast (iDPC) STEM image of the GaN on nitrogen-plasma-treated graphene. The arrangement of $\mathrm{Ga}$ and $\mathrm{N}$ atoms indicates that the $\mathrm{GaN}$ film grown on nitrogen-plasma-treated graphene is $\mathrm{Ga}$ polarity.

To determine the macroscopic orientation and crystal structure of $\mathrm{GaN}$ films on nitrogen-plasma-treated graphene, we also performed XRD measurements on GaN films. The XRD $2 \theta$ and $\varphi$ scan results show that GaN film on graphene is a well-aligned single crystal with hexagonal wurtzite structure (see Fig. S4). The improvement of GaN nucleation on graphene not only reduces the surface roughness of overgrown $\mathrm{GaN}$, but also improves its crystalline quality, as shown in Fig. 6a, b. The full-width at half-maximum (FWHM) of (0002) and (1012) planes rocking curve of $\mathrm{GaN}$ epilayer are greatly reduced from 1260 and 1440 arcsec to 232 and 290 arcsec, respectively. The crystalline quality of $\mathrm{GaN}$ films on graphene is comparable to that of $\mathrm{GaN}$ films grown directly on other conventional foreign substrates, such as $\mathrm{Si}$ and sapphire (see Table S1).

The stress in as-grown GaN films is further evaluated by Raman spectroscopy. The $E_{2}$ (high) phonon mode in Raman spectra is sensitive to the biaxial strain of $\mathrm{GaN}$ films, and therefore it can be used to evaluate the biaxial stress in III-nitrides ${ }^{26}$. For stress-free GaN bulk materials, 

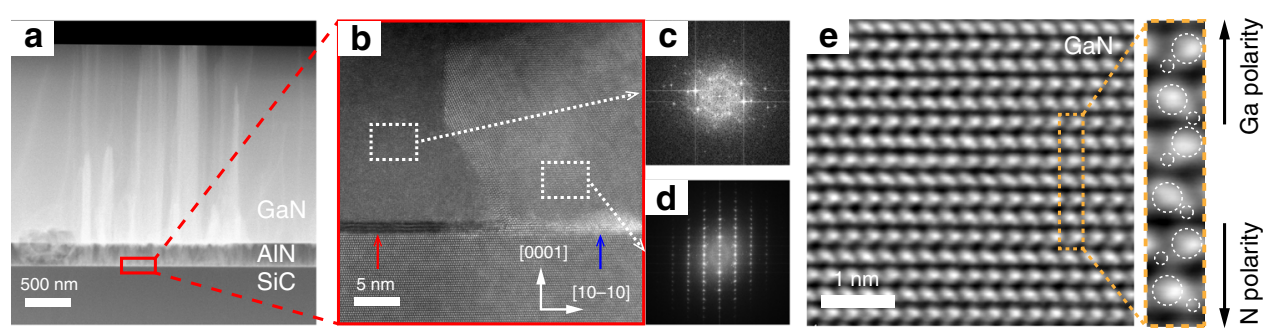

Fig. 5 HAADF-STEM analysis of GaN on nitrogen-plasma-treated graphene/SiC. a Cross-sectional HAADF-STEM image of GaN on nitrogenplasma-treated graphene. $\mathbf{b}$ Cross-sectional HAADF-STEM image of the interface area marked with a red rectangle frame in (a). The red arrow points to site where the dark part of the unetched graphene, whereas the blue arrow points to site where the light part of the etched graphene. $\mathbf{c}$, $\mathbf{d}$ SAED patterns of AIN on the graphene/SiC marked with a white rectangular frame in (b). e iDPC-STEM image of GaN grown on nitrogen-plasma-treated graphene/SiC. The atomic arrangement of Ga and N atoms confirms the Ga-polarity for the as-grown GaN
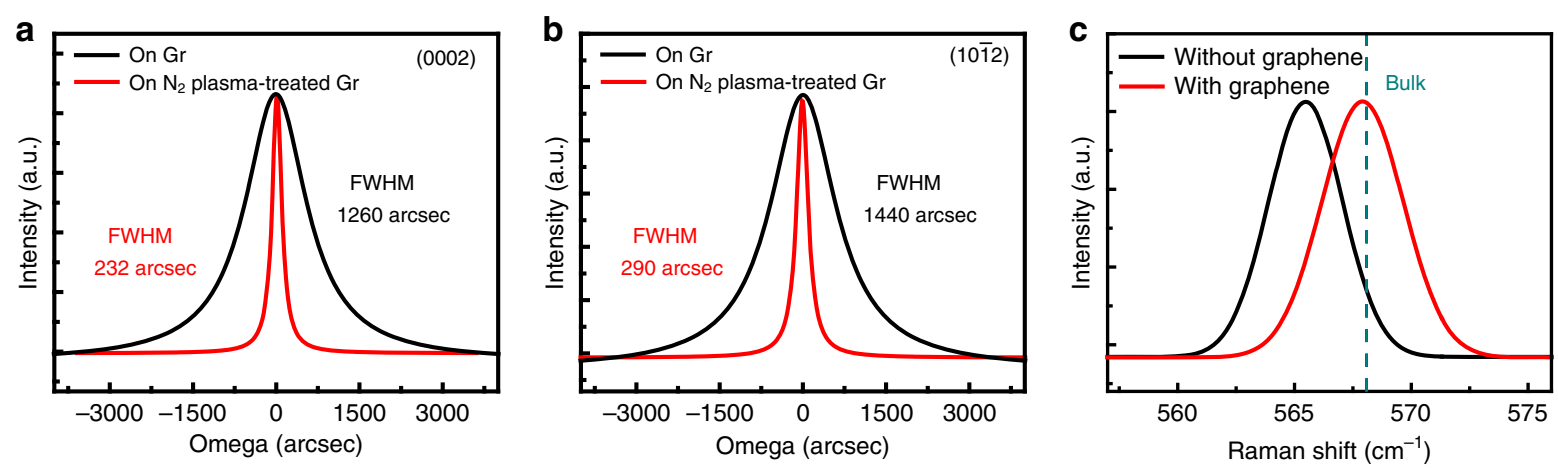

Fig. 6 Characterizations of GaN films on graphene/SiC. X-ray rocking curves of: a (0002), and $\mathbf{b}(10 \overline{2} 2)$ planes for $2 \mu \mathrm{m}$ thick GaN films grown on untreated graphene/SiC and nitrogen-plasma-treated graphene/SiC substrates. c Raman spectra of GaN films epitaxially grown on graphene (red) and directly on SiC substrate (black). The green dashed line is the $E_{2}$ (high) phonon frequency of GaN bulk material under stress-free state

its $\mathrm{E}_{2}$ (high) phonon frequency is $568 \mathrm{~cm}^{-127}$. Figure $6 \mathrm{c}$ shows that the $\mathrm{E}_{2}$ (high) phonon frequencies of GaN films grown on graphene and directly on $\mathrm{SiC}$ are both less than $568 \mathrm{~cm}^{-1}$, indicating that both GaN films are under tensile stress state $^{28}$. While, the $\mathrm{E}_{2}$ (high) phonon frequency of $\mathrm{GaN}$ on nitrogen-plasma-treated graphene is closer to that of stress-free $\mathrm{GaN}$, which is $567.9 \mathrm{~cm}^{-1}$. The specific biaxial stress value $\sigma$ of $\mathrm{GaN}$ epilayers can be calculated by the formula: $\sigma=\Delta \omega / \kappa$, where $\kappa$ is the stress coefficient, $\Delta \omega$ is the Raman frequency shift relative to the $\mathrm{E}_{2}$ (high) phonon frequency of stress-free GaN films. Here, $k=$ $-3.4 \mathrm{~cm}^{-1} \mathrm{GPa}^{-1}$ is adopted for the calculation ${ }^{29}$. The calculated residual tensile stress value for the GaN films on graphene is only $0.03 \mathrm{GPa}$, which is much lower than that of $\mathrm{GaN}$ films grown directly on $\mathrm{SiC}$ substrate $(0.74 \mathrm{GPa})$. This result shows that the residual stress in $\mathrm{GaN}$ films can be significantly reduced by inserting graphene.

Based on the above results, we propose a growth model to explain the growth mechanism of GaN films on graphene. As shown in Fig. 7a, AlN nucleation islands grown directly on untreated graphene exhibit a random in-plane orientation. After nitrogen-plasma pre-treatment on graphene, part of its surface area is etched, as shown in Fig. 7c. At the same time, $\mathrm{C}-\mathrm{N}$-related dangling bonds are formed on graphene surface. In Fig. 7d, the orientation of AlN on etched graphene region continues the orientation of $\mathrm{SiC}$ substrate, showing a single crystalline $c$-axis orientation growth. The $c$-axis-oriented AlN nucleation islands gradually occupy the dominant role, and a single crystal AlN layer with the same orientation is formed by lateral epitaxy. The subsequent $\mathrm{GaN}$ films are grown on the AlN layer, as shown in Fig. 7e. From the crosssectional HAADF-STEM image, we can see the grain boundaries generated during the lateral merging of AlN above different regions of graphene, as shown in Fig. 7f, which further verifies the rationality of the proposed growth model.

Based on above results, InGaN/GaN MQWs were grown on the as-grown $\mathrm{GaN} /$ graphene/ $\mathrm{SiC}$ template. For comparison, we also prepared InGaN/GaN MQWs with the same structure on $\mathrm{GaN} / \mathrm{SiC}$ template under the same conditions. The two samples were grown in one run. Figure $8 \mathrm{a}$, b exhibit XRD $2 \theta$ scan spectra for InGaN/GaN MQWs, where intense diffraction peaks from $\mathrm{GaN}$ epilayer and satellite peaks from the InGaN/GaN MQWs up 


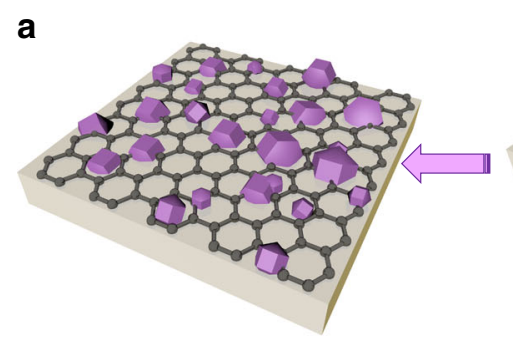

f

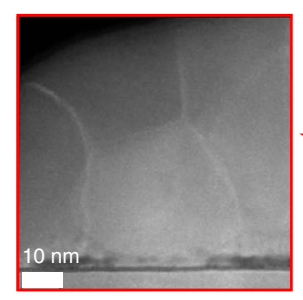

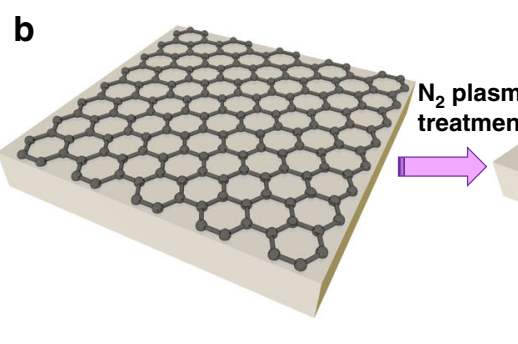

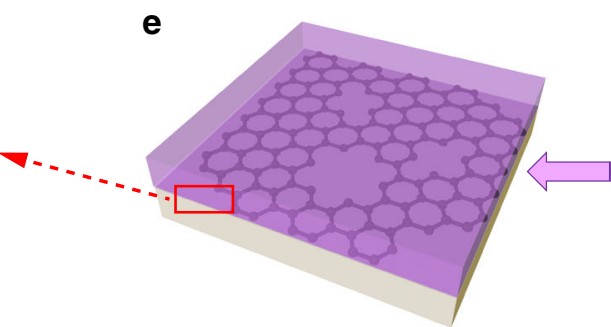

C

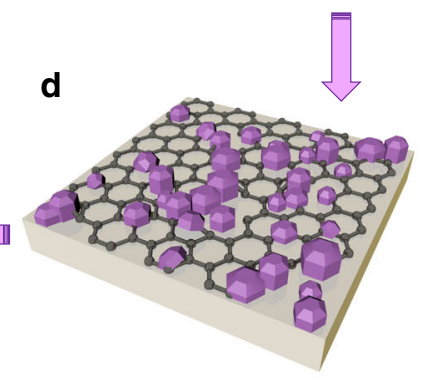

Fig. 7 Schematic diagram of the growth model of GaN films on nitrogen-plasma-treated graphene. a AIN nucleation islands on untreated graphene. The nucleation orientation shows a random in-plane orientation. $\mathbf{b}$ Direct growth of graphene on SiC substrate. $\mathbf{c}$ As-grown graphene after nitrogen-plasma pre-treatment. The $\mathrm{C}-\mathrm{N}$-related dangling bonds were formed on nitrogen-plasma-treated graphene, and the gray, red, and blue spheres represent the $\mathrm{C}$, pyrrolic $\mathrm{N}$, and pyridinic $\mathrm{N}$ atoms, respectively. $\mathbf{d}$ AIN nucleation islands on nitrogen-plasma-treated graphene. The nucleation orientation of AIN on the etched graphene region continues the orientation of SiC substrate, which is a single crystal with c-axis orientation. e Epitaxial growth of continuous GaN films on AIN buffer. $\mathbf{f}$ Cross-sectional HAADF-STEM image of the interface at AIN/graphene/SiC
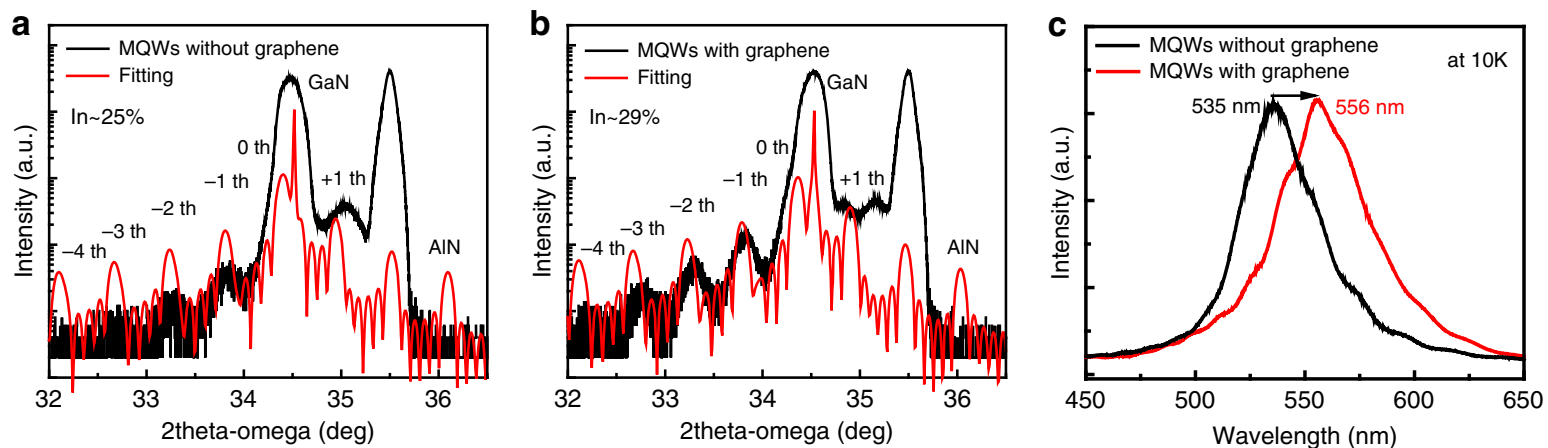

Fig. 8 Characterizations of InGaN/GaN MQWs on SiC and graphene/SiC substrates, respectively. XRD 2O- $\omega$ scans spectra of (0002) plane for InGaN/GaN MQWs grown on: a SiC, and b graphene/SiC substrates, respectively. c PL spectra of InGaN/GaN MQWs grown on SiC and graphene/SiC substrates $(T=10 \mathrm{~K})$

to the fourth order can be observed in both samples, indicating good crystalline quality and sharp interfaces of InGaN/GaN MQWs. From the fitting results, it can be found that the indium (In) content in InGaN well layer increases from $25 \%$ to $29 \%$ after inserting graphene. Figure 8c shows the PL spectra of InGaN/GaN MQWs at a low temperature of $10 \mathrm{~K}$, which indicates that the insertion of graphene results in a red-shift of MQWs emission wavelength from 535 to $556 \mathrm{~nm}$, and the shift is about $21 \mathrm{~nm}$. Based on the peak positions of the PL spectra, the calculated In content in the InGaN/GaN MQWs on graphene/ $\mathrm{SiC}$ is $3.5 \%$ higher than that on $\mathrm{SiC}$, which is close to the XRD fitting results (In content increased by 4\%). According to the Raman results, it is found that the stresses are greatly different in the $\mathrm{GaN}$ templates on graphene/ $\mathrm{SiC}$ and $\mathrm{SiC}$. The residual stress in the $\mathrm{GaN}$ template on graphene/ $\mathrm{SiC}$ is much lower than that on SiC. Therefore, we think that the strain relaxation is the main reason for the increase of In content in InGaN/GaN MQWs.

\section{Discussion}

In conclusion, we realized the epitaxy of strain-relaxed $\mathrm{GaN}$ films on graphene/SiC substrates. Graphene was directly prepared on $\mathrm{SiC}$ substrates by thermal decomposition. The pre-treatment of graphene with nitrogen-plasma 
can generate $\mathrm{N}-\mathrm{sp}^{3} \mathrm{C}$ dangling bonds, which facilitate the nucleation and growth of subsequent epitaxy. STEM results provide direct evidence that the surface of graphene is partially etched by nitrogen-plasma pre-treatment. Based on the growth behavior on different etched areas of graphene surface after pre-treatment, a growth model is proposed to explain the epitaxial growth mechanism of GaN films on graphene. It is worth noting that, graphene can effectively reduce the biaxial stress in $\mathrm{GaN}$ films and the strain relaxation improves the incorporation efficiency of In atoms in InGaN/GaN MQWs. This work provides a feasible way for the epitaxy of strain-relaxed GaN films and opens up a new avenue for the fabrication of GaN-based long wavelength LEDs.

\section{Materials and methods Preparation of graphene}

Graphene was prepared on $\mathrm{Si}$-face $4 \mathrm{H}-\mathrm{SiC}$ substrates by thermal decomposition. Firstly, $4 \mathrm{H}-\mathrm{SiC}$ substrates were annealed in an Ar atmosphere at $800-1000^{\circ} \mathrm{C}$ for $1 \mathrm{~h}$ to remove scratches and oxygen on the surface of $\mathrm{SiC}$ substrate. Then, $\mathrm{SiC}$ substrates was heated to $1600-1700^{\circ} \mathrm{C}$ in an ultra-high purity Ar atmosphere under 800 mbar for $2-5 \mathrm{~h}$ to realize surface graphitization.

\section{Epitaxial growth of GaN films and InGaN/GaN MQWs}

$\mathrm{GaN}$ films were grown on graphene/SiC substrates by an AIXTRON $3 \times 2^{\prime \prime}$ FT MOCVD system. Trimethylgallium (TMGa), trimethylaluminum (TMAl), and ammonia $\left(\mathrm{NH}_{3}\right)$ were used as $\mathrm{Ga}, \mathrm{Al}$, and $\mathrm{N}$ precursors, respectively. $\mathrm{H}_{2}$ and $\mathrm{N}_{2}$ were used as carrier gases. Prior to the deposition, graphene/ $\mathrm{SiC}$ substrates were thermally cleaned in $\mathrm{H}_{2}$ atmosphere at $1100{ }^{\circ} \mathrm{C}$ for $5 \mathrm{~min}$. The growth began with the low-temperature AlN buffer layer of about $40 \mathrm{~nm}$ thickness grown at $780^{\circ} \mathrm{C}$. Then, the growth temperature ramped up to $1080^{\circ} \mathrm{C}$ for the growth of high-temperature AlN buffer layer with thickness of about $160 \mathrm{~nm}$. Subsequently, a $2 \mu \mathrm{m}$ thick undoped GaN layer was grown at $1050^{\circ} \mathrm{C}$. After that, the InGaN/GaN MQWs were grown on the undoped GaN layer. The MQWs consist of 5 pairs of $2.5 \mathrm{~nm}$ thick InGaN quantum well (QW) layers and $15 \mathrm{~nm}$ thick GaN quantum barrier (QB) layers. QW and QB layers were grown at 712 and $840^{\circ} \mathrm{C}$, respectively, and they were undoped. For comparison, InGaN/GaN MQWs without graphene interlayer were fabricated directly on $\mathrm{SiC}$ substrate. It is worth noting that these two kinds of MQWs samples were grown in one run.

\section{Characterization}

The surface morphology of graphene and GaN films were measured by SEM (Jeol-7500F) and AFM in tapping mode (Bruker Dimension ICON-PT). Raman spectroscopy (Renishaw inVia, $532 \mathrm{~nm}$ laser excitation) was performed to characterize the quality of graphene and the stress state of GaN films. The chemical states of graphene were measured by an ESCALab 250 Analytical XPS spectrometer with a monochromatic X-ray source $(\mathrm{Al} \mathrm{K \alpha}$, $\mathrm{h} v=1486.6 \mathrm{eV})$. The binding energies of the spectra were referred to that of the $\mathrm{C} 1 \mathrm{~s}$ peak at $\sim 284.8 \mathrm{eV}$. The crystalline quality of $\mathrm{GaN}$ films characterized with a Rigaku Ultima IV XRD with $\mathrm{Cu} \mathrm{K} \alpha$ radiation $(0.154056 \mathrm{~nm})$, and the structural properties of GaN films were investigated by aberration corrected Thermo Fisher Scientific Titan Cubed Themis G2 transmission electron microscope operated at $300 \mathrm{kV}$ and equipped with Bruker Super-X EDX detector. The PL spectra of InGaN/GaN MQWs were measured by a PL system which includes a $\mathrm{He}-\mathrm{Cd}$ laser $(325 \mathrm{~nm}, 30 \mathrm{~mW})$ as an excitation source, a Jobin Yvon iHR550 spectrometer, a Syncerity charge coupled device (CCD) and a closed circle helium cryostat.

\section{Acknowledgements \\ This work was supported by the National Key Research and Development Program (No. 2018YFB0406703), the National Natural Science Foundation of China (Nos. 61734001 and 62074069), the Science and Technology Developing Project of Jilin Province (No. 20200801013GH), and the Program for JLU} Science and Technology Innovative Research Team (JLUSTIRT, 2021TD-39).

\section{Author details}

${ }^{1}$ State Key Laboratory of Integrated Optoelectronics, College of Electronic Science and Engineering, Jilin University, Qianjin Street 2699, Changchun 130012, China. ${ }^{2}$ Electron Microscopy Laboratory, School of Physics, Peking University, Beijing 100871, China. ${ }^{3}$ State Key Laboratory of Crystal Materials, Shandong University, Jinan 250100, China. ${ }^{4}$ State Key Laboratory for Mesoscopic Physics and Frontiers Science Center for Nano-optoelectronics, School of Physics, Peking University, Beijing 100871, China. ${ }^{5}$ Key Laboratory of Materials Physics of Ministry of Education, School of Physics and

Microelectronics, Zhengzhou University, Zhengzhou 450052, China

Conflict of interest

The authors declare no competing interests.

Supplementary information The online version contains supplementary material available at https://doi.org/10.1038/s41377-021-00560-3.

Received: 7 February 2021 Revised: 13 May 2021 Accepted: 21 May 2021 Published online: 03 June 2021

\footnotetext{
References

1. Meng, X. et al. Study on efficiency droop in InGaN/GaN light-emitting diodes based on differential carrier lifetime analysis. Appl. Phys. Lett. 108, 013501 (2016).

2. Martens, M. et al. Low absorption loss p-AIGaN superlattice cladding layer for current-injection deep ultraviolet laser diodes. Appl. Phys. Lett. 108, 151108 (2016).

3. Bao, G. H. et al. Enhanced spectral response of an AlGaN-based solar-blind ultraviolet photodetector with Al nanoparticles. Opt. Express 22, 24286-24293 (2014).

4. Zhang, Y. T. et al. Demonstration of N-polar III-nitride tunnel junction LED. ACS Photonics 7, 1723-1728 (2020).

5. Deng, G. Q. et al. Significantly improved surface morphology of N-polar GaN film grown on SiC substrate by the optimization of V/III ratio. Appl. Phys. Lett 112, 151607 (2018).

6. Jiang, F. Y. et al. Efficient InGaN-based yellow-light-emitting diodes. Photonics
} Res. 7, 144-148 (2019). 
7. Zhao, D. G. et al. Stress and its effect on optical properties of GaN epilayers grown on Si(111), 6H-SiC (0001), and c-plane sapphire. Appl. Phys. Lett. 83, 677-679 (2003).

8. Schubert, M. F. et al. Effect of dislocation density on efficiency droop in InGaN/GaN light-emitting diodes. Appl. Phys. Lett. 91, 231114 (2007).

9. Zollner, C. J. et al. Reduced dislocation density and residual tension in AIN grown on SiC by metalorganic chemical vapor deposition. Appl. Phys. Lett. 115, 161101 (2019).

10. Chung, K., Lee, C. H. \& Yi, G. C. Transferable GaN layers grown on ZnO-Coated graphene layers for optoelectronic devices. Science 330, 655-657 (2010).

11. Liu, F. et al. Graphene-assisted epitaxy of nitrogen lattice polarity GaN films on non-polar sapphire substrates for green light emitting diodes. Adv. Funct. Mater. 30, 2001283 (2020).

12. Liu, F. et al. Hexagonal BN-assisted epitaxy of strain released GaN films for true green light-emitting diodes. Adv. Sci. 7, 2000917 (2020).

13. Susanto, I. et al. The influence of $2 \mathrm{D} \mathrm{MoS}$ layers on the growth of $\mathrm{GaN}$ films by plasma-assisted molecular beam epitaxy. Appl. Surf. Sci. 496, 143616 (2019).

14. Kim, Y. et al. Remote epitaxy through graphene enables two-dimensional material-based layer transfer. Nature 544, 340-343 (2017).

15. $Y u, J$. D. et al. Study on AIN buffer layer for GaN on graphene/copper sheet grown by MBE at low growth temperature. J. Alloy. Compd. 783, 633-642 (2019).

16. Kim, J. et al. Principle of direct van der Waals epitaxy of single-crystalline films on epitaxial graphene. Nat. Commun. 5, 4836 (2014)

17. Gupta, P. et al. Free-standing semipolar III-nitride quantum well structures grown on chemical vapor deposited graphene layers. Appl. Phys. Lett. 103 181108 (2013)
18. Chen, Z. L. et al. Improved epitaxy of AIN film for deep-ultraviolet light-emitting diodes enabled by graphene. Adv. Mater. 31, 1807345 (2019).

19. Lee, S. et al. Proton irradiation energy dependence of defect formation in graphene. Appl. Surf. Sci. 344, 52-56 (2015).

20. Mathew, S. et al. The effect of layer number and substrate on the stability of graphene under MeV proton beam irradiation. Carbon 49, 1720-1726 (2011).

21. Kim, J. et al. Layer-resolved graphene transfer via engineered strain layers. Science 342, 833-836 (2013).

22. Kong, $W$. et al. Polarity governs atomic interaction through two-dimensional materials. Nat. Mater. 17, 999-1004 (2018).

23. Cancado, L. G. et al. Quantifying defects in graphene via Raman spectroscopy at different excitation energies. Nano Lett. 11, 3190-3196 (2011).

24. Al Balushi, Z. Y. et al. The impact of graphene properties on GaN and AIN nucleation. Surf. Sci. 634, 81-88 (2015).

25. Reddy, A. L. M. et al. Synthesis of nitrogen-doped graphene films for lithium battery application. ACS Nano 4, 6337-6342 (2010).

26. Park, A. H. et al. Efficient stress-relaxation in InGaN/GaN light-emitting diodes using carbon nanotubes. Nanoscale 7, 15099-15105 (2015).

27. Perlin, $\mathrm{P}$. et al. Raman scattering and X-ray-absorption spectroscopy in gallium nitride under high pressure. Phys. Rev. B 45, 83-89 (1992).

28. Kisielowski, C. et al. Strain-related phenomena in GaN thin films. Phys. Rev. B 54 17745-17753 (1996)

29. Ahmad, I. \& Holtz, M. Dependence of the stress-temperature coefficient on dislocation density in epitaxial $\mathrm{GaN}$ grown on $\mathrm{a}-\mathrm{Al}_{2} \mathrm{O}_{3}$ and $6 \mathrm{H}-\mathrm{SiC}$ substrates. J. Appl. Phys. 95, 1692-1697 (2004). 